\title{
Connection of Collimation, Asymmetric Beaming, and Independent Transmission-Reflection Processes in Concentric-Groove Gratings Supporting Spoof Surface Plasmons
}

\author{
Mohsin Habib 1,2,3 (D) Andriy E. Serebryannikov ${ }^{4,5}$ • Humeyra Caglayan ${ }^{3}$. Guy A. E. Vandenbosch ${ }^{4}$. \\ Ekmel Ozbay ${ }^{1,2,6,7}$
}

Received: 18 July 2018 / Accepted: 27 August 2018 / Published online: 7 September 2018

(C) Springer Science+Business Media, LLC, part of Springer Nature 2018

\begin{abstract}
Transmission through subwavelength apertures enables separation of the incidence half-space and the exit half-space, which leads to that the spatial distribution of the field in the latter is not affected by the distribution in the former. The distribution in the exit half-space is mainly determined by the properties of surface plasmons (SPs) at the exit-side interface. In this paper, for the microwave structures with one-side concentric corrugations around a single annular hole, we demonstrate the possible connections between asymmetric transmission in the beaming regime and collimation of the waves incident at different angles, which can be considered as two sides of the same phenomenon occurring due to the common effect of such a separation and the radiation shaping effect being possible due to the spoof SPs at the corrugated exit interface. Collimation manifests itself in that the waves incident at different angles from a wide range contribute to the single outgoing beam so that a far-zone observer cannot distinguish between the contributions of different angles of arrival. Asymmetry in transmission manifests itself in that the spatial shaping of radiation (beaming) in the exit half-space appears only for one of the two opposite incidence directions. Moreover, even in the structures with the same corrugations on both sides, i.e., without asymmetric transmission, spatial separation of two wave processes, e.g., two symmetric or asymmetric collimation processes, can be obtained for a wide range of nonzero angles of incidence.
\end{abstract}

Keywords Collimation $\cdot$ Beaming $\cdot$ Asymmetric transmission $\cdot$ Spoof plasmon

\section{Introduction}

The capability of electrically small apertures, i.e., holes and slits in transmission, which is known as the extraordinary transmission phenomenon, has been the focus of research in the last two decades [1]. A variety of the physical scenarios connected with extraordinary transmission has

Mohsin Habib

mohsin.habib@bilkent.edu.tr

1 Department of Electrical and Electronics Engineering, Bilkent University, 06800, Ankara, Turkey

2 NANOTAM-Nanotechnology Research Center, Bilkent University, 06800, Ankara, Turkey

3 Laboratory of Photonics, Tampere University of Technology, 33720, Tampere, Finland been demonstrated for both single holes/slits and their arrays in a wide range that extends from microwave to optical frequencies. Transmission through subwavelength apertures can be enhanced or vanishing, depending on the properties of the surrounding corrugations [2-5]. Generally, transmission in these structures can be considered as the consequence of three independent processes: in-coupling,

4 ESAT-TELEMIC, Katholieke Universiteit Leuven, 3000, Leuven, Belgium

5 Faculty of Physics, Adam Mickiewicz University, 61-614, Poznan, Poland

6 Department of Physics, Bilkent University, 06800, Ankara, Turkey

7 UNAM-National Institute of Materials Science and Nanotechnology, Bilkent University, 06800, Ankara, Turkey 
transmission through, and out-coupling [6]. It has been shown that (spoof or designer) surface plasmons (SPs) excited at the incidence interface are responsible for the transmission enhancement. At the same time, the (spoof) SPs excited at the exit interface are mainly responsible for the field distribution in the exit half-space, e.g., for the shaping of the outgoing beam [7, 8]. In such a way, incidence and exit half-spaces can be separated, i.e., field distribution in one of them is not affected by the other, in spite of that the structure is electrically thin. In one of our previous works, we have shown that by coupling SPs and incident wavelengths using circular aperture, the enhancement in transmission can be achieved [9].

In the structures with a long subwavelength slit and grooves parallel to it, by properly engineering lamellar corrugations at the left side of the exit interface and at the right side of the exit interface, or placing proper corrugations only at the left or the right side, one can obtain off-axis beaming [10-14]. When different corrugations are placed at the left and the right side, the (spoof) SPs can be excited so that the both sides contribute into a single beam in the off-axis regime [10, 12, 13]. These regimes are often considered a manifestation of collimation. A similar phenomenon was reported for acoustic surface waves, in which multiple input wavelengths contribute to single outgoing beam [15]. Strictly speaking, when the incident angle is the same for the waves of different frequencies and a single beam is collected, one obtains multiplexing rather than collimation. In turn, for collimation, we expect that contributions of different incident waves at the same frequency but different incident angles are collected at single wave or beam [16]. This can happen when (spoof) SPs excited by the waves incident at different angles have (very) similar properties. On the other hand, by using a single incident wave and engineered nonuniform corrugations, one can obtain energy focusing at a particular focal point, while SPs excited at individual segments of this structure are different [17]. A similar regime can be obtained in the structures with uniform corrugations, which are either different or identical at the left and the right side [11].

Spatial separation of the incidence and the exit halfspace due to a subwavelength aperture may enable one more interesting phenomenon-diodelike asymmetric transmission. Asymmetric transmission (AT) manifests itself in that the difference in transmission is changed dramatically when the incidence direction is changed for the opposite one, provided that the spatial inversion symmetry of the structure is broken. In particular, this enables diodelike asymmetry in transmission in the beaming regime. Generally speaking, AT includes a wide class of transmission scenarios, which are realizable in various nonsymmetric structures like photonic-crystal gratings [1820], metamaterial-based gratings [21, 22], (ultra-)thin gratings with subwavelength slit(s) [16, 26, 27], gratings made of epsilon-near-zero materials [28], photonic-crystal prisms [29, 30], and metasurfaces [31-33]. AT can be explained in terms of the differences of mode coupling and mode conversion at front-side and back-side illumination. In thin gratings with a single subwavelength slit, AT may appear in the beaming regime, if the (spoof) SPs at the exit interface enable beam shaping [16, 34, 35].

In the structures with a hole, with different functionalities including AT, collimation, and others, one may choose between the classical geometry (circular hole surrounded by concentric grooves) and more complex geometries. For instance, generation of Bessel beams in the structures with a rectangular hole surrounded by double-periodic corrugations can be mentioned [23]. Moreover, nonconcentric grooves can be used to obtain off-axis radiation, as has been recently demonstrated in millimeter-wave [24] and optical [25] bull's-eye antennas. Annular apertures are known for their capability to enhance transmission as compared with circular apertures of the same radius [3, 36, 37]. Indeed, this allows utilizing the waves propagating in the transmission channel(s) connecting the incidence and the exit half-space, instead of the evanescent waves. However, until now, possible effects of annular apertures on collimation of the outgoing radiation and AT have not been studied.

In this paper, we study transmission through a single annular hole in a thin metallic screen, which is surrounded with the grating created by equidistant coaxial grooves at one side or both sides of the structure. Consideration is restricted to the microwave frequency range. After a short discussion of the effect of size of the illuminated area (i.e., the effect of the number of contributing concentric grooves) and inner diameter of the annular hole, we will focus on the study of transmission for the structure with one-side grooves (corrugations), in a wide range of the incidence angle that extends from 0 to $75^{\circ}$. The first goal of this study is to analyze the capability of the studied structure in collimation of multiple waves incident at different angles into a single outgoing beam. The next goals are to demonstrate the appearance of AT and its possible connection to wide-angle collimation in nonsymmetric structures with a single hole. It will be shown that AT and collimation represent two sides of the same phenomenon. Then, we discuss the specifics of the structures with twoside corrugations. In particular, it will be shown how two (groups of) independent incidence-transmission-reflection processes connected with collimation can co-exist in such a structure. For the purposes of the numerical study, CST Microwave Studio, a commercial software based on finiteintegration method has been used (https://www.cst.com). The studied structures are assumed to be made of aluminum, whose conductivity is taken from the material library of CST Microwave Studio. 


\section{SP-Assisted Transmission Through a Single Annular Hole}

SPs play important role in transmission through subwavelength apertures. Canonical SPs on flat metal-dielectric interfaces represent collective excitation of the surface electrons in a metal, which satisfies the following condition:

$k_{s p}=k_{0} \sqrt{\frac{\epsilon_{m} \epsilon_{d}}{\epsilon_{m}+\epsilon_{d}}}$,

where $k_{0}$ is incident wave vector portion in metal plane, $\epsilon_{m}$ and $\epsilon_{d}$ are permittivity of metal and dielectric. However, they do not interact with the incident light. Placing corrugations at the interfaces can lead to desired interaction. Moreover, the surface waves known as spoof SPs, which are not identical but strongly similar to the canonical SPs, may exist at the corrugated interfaces even in the perfect electrical conductor (PEC) approximation, i.e., at the operating frequencies being much smaller than those ones, at which the canonical SPs could exist. This remains true for a very wide class of the structures, which include one-dimensional arrays of the grooves and two-dimensional hole arrays [38].

Transmission through the subwavelength apertures, either representing an array themselves or being surrounded by the additional corrugations, have been studied widely over the last decades, as the (spoof) SPs arising due to corrugations can be coupled and provide transmission enhancement and efficient collection of electromagnetic radiation [39-42]. Most of the studies have been done for the circular apertures. Baida et al. have earlier found that an annular aperture may provide a stronger transmission enhancement compared to a circular aperture, since it is created by a section of the coaxial waveguide supporting waveguide modes, including a transverse electromagnetic (TEM) mode without cutoff $[36,43]$. However, excitation of a TEM mode by a linearly polarized incident wave is not possible. Thus, transverse electric (TE) and transverse magnetic (TM) modes are required to obtain transmission. The general form of dispersion relation for these modes can be written as follows:

$k_{z}=\frac{4 \pi^{2}}{\lambda}-\frac{4 \pi^{2}}{\lambda_{c}}$,

where $\lambda_{c}$ is the cutoff wavelength. For TE modes, which are expected to provide a desired transmission, $\lambda_{c}$ is given by

$\lambda_{c T E m, 1} \approx \frac{\pi(a+b)}{2 m}$.

Here, $a$ and $b$ are inner and outer diameters of the annular aperture, respectively. Combination of the annular aperture with exit-side corrugations, as shown in Fig. 1, is known to result in enhancement of extraordinary transmission into and beaming in the exit half-space, which are highly
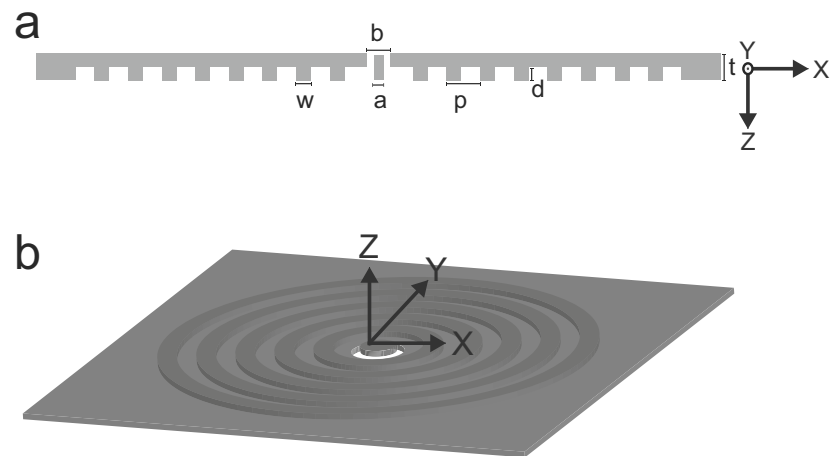

Fig. 1 Schematic of annular hole surrounded by uniform corrugations. a Cross section. b Perspective view

demanded in the practical devices [9]. It is seen in Eq. 3 that $\lambda_{c T E m, 1}$ is proportional to $a+b$. Illumination is modeled by a CST waveguide port, with E-field vector being along $y$ axis and $\mathrm{H}$-field vector being along $x$-axis (www.cst.com). This model approximately corresponds to the illumination by a horn antenna.

In our study, the grating thickness, $t$, was varied from $5.5 \mathrm{~mm}$ to $20 \mathrm{~mm}$. The results are presented for the case of $b=t=8 \mathrm{~mm}$ throughout the paper. The emphasis is put on the case of $a=6.5 \mathrm{~mm}$, for which the strong effect of the spoof SPs on transmission and shaping of the outgoing radiation occurs near $13 \mathrm{GHz}$. Transmission and field behavior at other frequencies have also been checked (not shown).

The value of $a$ affects the cutoff wavelength, strength of coupling, and thus capability of beaming. Effect of the inner diameter is demonstrated in Fig. 2. Since transmission through the annular aperture occurs due to the effect of a propagating mode, transmission efficiency can remain high at rather arbitrary thicknesses which are limited by Ohmic losses in aluminum. Transmission is insignificant in the absence of inner rod, see Fig. 2a, e. Introducing a rod with diameter of $a=4 \mathrm{~mm}$ results in that the transmission is to somehow enhanced but still remains low in the exit halfspace, as shown in Fig. 2b, f. As $a$ is increased to $5.2 \mathrm{~mm}$ and then to $6.5 \mathrm{~mm}$, transmission in the beaming regime is enhanced, compare Fig. 2c with Fig. 2d and Fig. 2g with Fig. $2 \mathrm{~h}$. Transmission becomes weaker at a further increase of $a$. The value of $a=6.5 \mathrm{~mm}$ is close to the optimal one for $13 \mathrm{GHz}$. It is noteworthy that the optimal value of $a$ tends to decrease while frequency is increased. For example, $a=5.75 \mathrm{~mm}$ could be used at $14 \mathrm{GHz}$.

As far as the spoof SPs occur in the plane perpendicular to the incident $\mathrm{H}$-field vector, their effect can be well seen from the comparison of Fig. $2 \mathrm{~g}$, h. Transmission in the beaming regime becomes stronger along with the spoof SPs, whose intensity depends, in turn, on the strengths of inand out-coupling and, thus, on the value of $a$. As has been mentioned above, corrugations at the incidence interface 

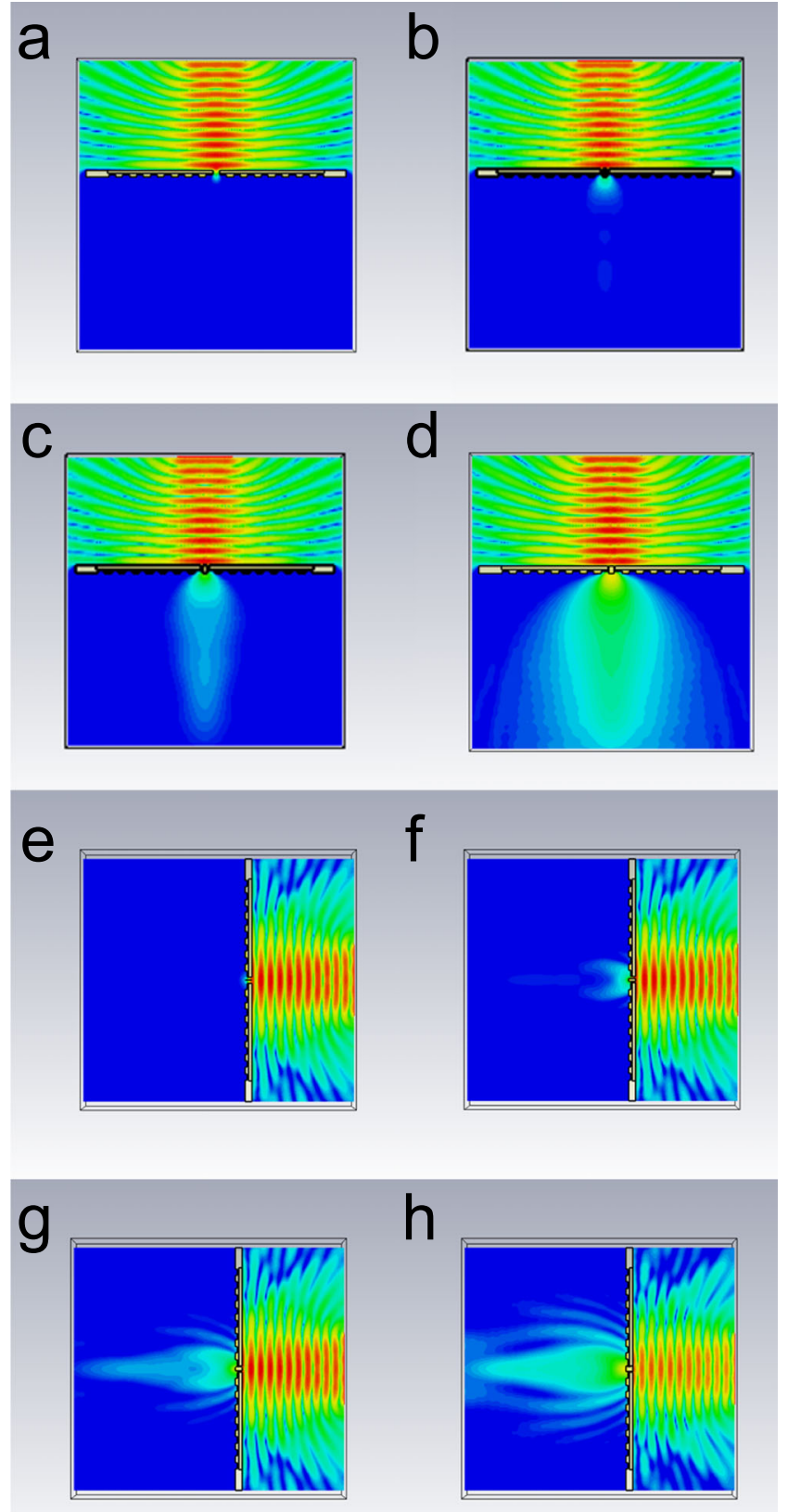

Fig. 2 Effect of rod diameter on field distribution at $f=13 \mathrm{GHz}$ : electric field in (x,z)-plane at $\mathbf{a} a=0, \mathbf{b} a=4 \mathrm{~mm}, \mathbf{c} a=5.2 \mathrm{~mm}$, and $\mathbf{d} a=6.5 \mathrm{~mm}$; magnetic field in $(z, y)$-plane at $\mathbf{e} a=0, \mathbf{f} a=4$ $\mathrm{mm}, \mathbf{g} a=5.2 \mathrm{~mm}, \mathbf{h} a=6.5 \mathrm{~mm} ; b=8 \mathrm{~mm}, t=8 \mathrm{~mm}, p=16 \mathrm{~mm}$, $d=2.4 \mathrm{~mm}$, and $w=p / 2$; noncorrugated-side illumination; all shown grooves are unfilled

may affect the transmission efficiency. At the same time, corrugations at the exit interface affect the shaping of the outgoing radiation. Figure 3 shows how this effect appears in the structure with one-side (exit-side) corrugations, when varying the number of the unfilled grooves around the annular aperture. Figure 3 presents the E-field magnitude

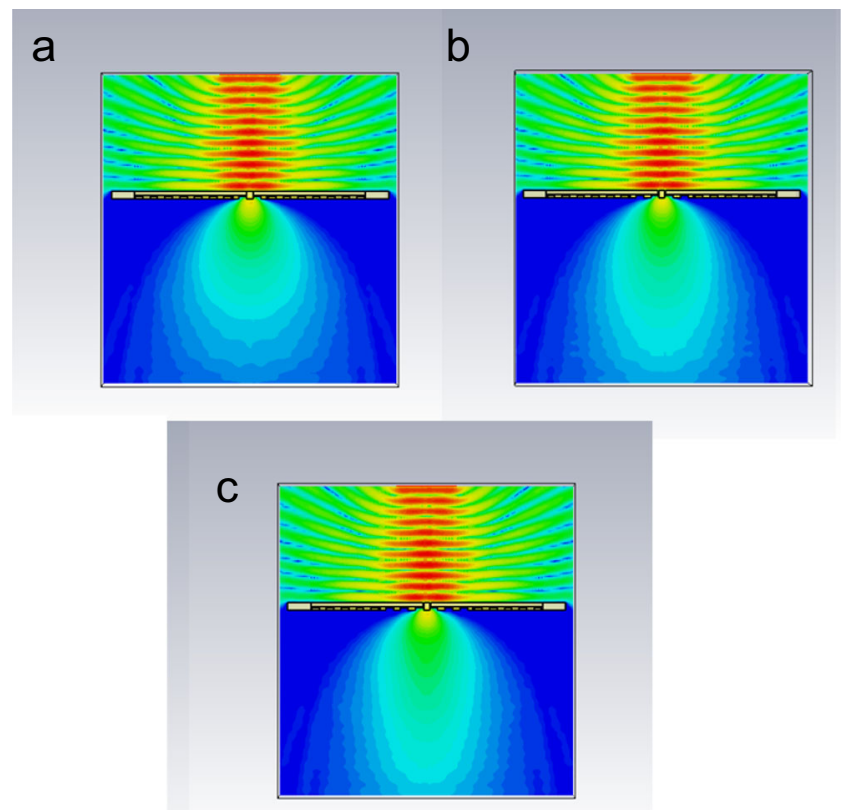

Fig. 3 Effect of increasing the exit-side grating extent on shaping. Electric field distribution at $f=13 \mathrm{GHz}$ for noncorrugated-side illumination. Only $\mathbf{a}$ one, $\mathbf{b}$ two, and $\mathbf{c}$ three concentric grooves around the hole are kept unfilled; the remaining grooves are assumed to be filled with metal; $b=8 \mathrm{~mm} ; t=8 \mathrm{~mm}, p=16 \mathrm{~mm}, d=2.4 \mathrm{~mm}$, and $w=p / 2$

when only one, two, and three grooves of the original structure in Fig. 2d are kept unfilled, while all other grooves are assumed to be filled with the metal (aluminum).

It is seen that no shaping effect occurs in the one-groove case and starts to manifest itself in the two-groove case, see Fig. 3a, b. In the three-groove case in Fig. 3c, the shaping of outgoing radiation takes place similarly to Fig. 2d. Indeed, there is no principal difference between the cases of three (Fig. 3c) and eight (Fig. 2d) grooves, i.e., only three grooves efficiently contribute to the resulting field distribution. This behavior can indicate a relatively short propagation length of spoof SPs in the studied structures. The role of three grooves being closest to the aperture can be clearly seen from the comparison with $\mathrm{H}$-field distribution in Fig. $2 \mathrm{~h}$. Nevertheless, in the next sections, we consider the structures with eight unfilled grooves, as in Fig. 2, in order to ensure that contributions of all parts of the exit interface are fully taken into account.

\section{Collimation and Asymmetry in Transmission}

In this section, we will demonstrate the separation of the incidence and exit half-spaces in sense of sensitivity of spatial field distribution, which occurs due to the common effect of an annular hole and spoof SPs. This can lead 

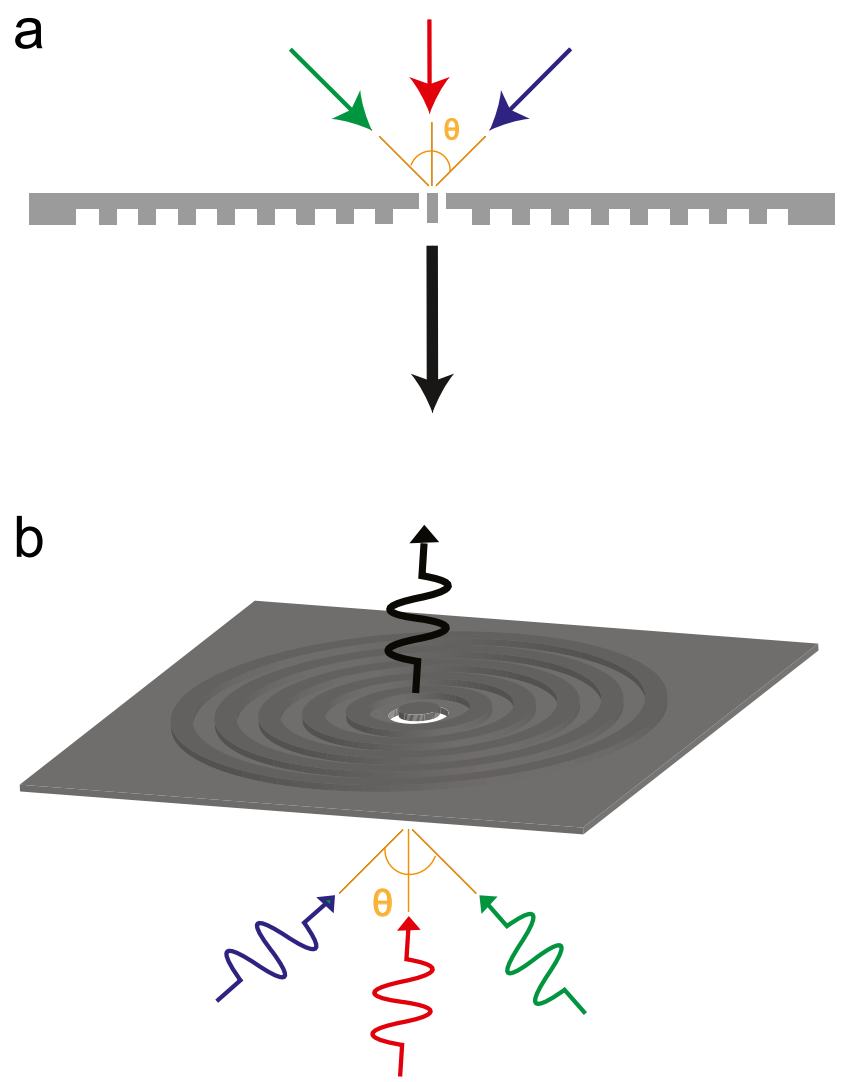

Fig. 4 Schematic illustrating the possible collimation of the waves incident at different angles on the structure with concentric grooves at the exit-side interface: a cross section; b perspective view. Contributions are expected to be collected from (a big part of) the whole cone

to collimation and asymmetric transmission, which are connected to each other. Figure 4 shows the schematic of the expected collimation in the structure with the exit-side corrugations and a central annular hole. The arrows in the incidence half-space indicate possible directions of incident beams, while the arrow in the exit half-space indicates the possible direction of the outgoing (collimated) beam.

In order to check our expectation regarding a possible contribution of different incident waves, which arrive at different angles, $\theta$, into the one outgoing beam, we carried out a detailed numerical study by varying $\theta$ from $0^{\circ}$ to $75^{\circ}$, with the step of $1^{\circ}$. The shaping of the outgoing radiation was observed for all of the $\theta$-values that indicate the strong and wide-angle effect of spoof SPs at the exit interface. As follows from the obtained results, the direction of the outgoing beam is the same for all of the values of $\theta$, so that it is not affected by the specifics of the field distribution in the incidence half-space at different values of $\theta$.

The illustrative examples are presented in Fig. 5 when the angle variation is achieved by rotating the structure around the $y$-axis. One can see that although the outgoing beam's
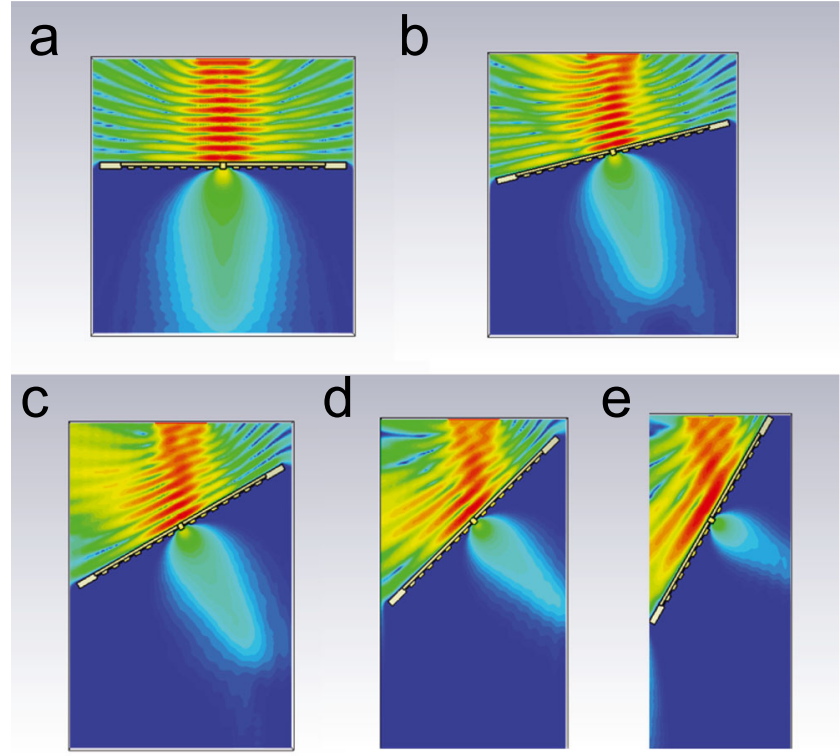

Fig. 5 Electric field distribution in ( $x, z)$-plane at $\mathbf{a} \theta=0^{\circ}, \mathbf{b} \theta=15^{\circ}$, c $\theta=30^{\circ}$, d $\theta=45^{\circ}$, e $\theta=60^{\circ} ; f=13 \mathrm{GHz}$, noncorrugatedside illumination; $a=6.5 \mathrm{~mm}, b=8 \mathrm{~mm} ; t=8 \mathrm{~mm}, p=16 \mathrm{~mm}$, $d=2.4 \mathrm{~mm}$, and $w=p / 2$

direction being normal to the interface is preserved, the intensity depends on $\theta$. This feature is connected with the fact that the coupling efficiency at the incidence interface depends on $\theta$. According to the superposition principle, several waves that are simultaneously incident at different angles and the same frequency will contribute to the same outgoing beam, i.e., collimation takes place. This means, among other things, that the angle(s) of arrival remain unknown for a far-field observer in the exit half-space, regardless of the number of the incident beams. In such a way, the location of the wave source(s) can be masked, at least if only magnitude information is available. The observed behavior can be useful for sensing applications, because contributions of the different arrival angles are accumulated by the one beam. Moreover, since the studied structure is azimuthally uniform, contributions of the waves for all possible values of $\theta$ can be accumulated. It is noteworthy that the beaming features are also observed at large $\theta$, e.g., at $\theta=60^{\circ}$ in Fig. 5e, and even at larger angles.

Next, we consider the case when the structure is rotated around the $x$-axis. Figure 6 presents $\mathrm{H}$-field magnitude at several values of the incidence angle. One can see that only a slight deviation of the outgoing beam from the direction normal to the interface (up to $3^{\circ}$ ) takes place, so that the ability to collect contributions from a wide range of the incidence angles is not affected.

Since breaking the structural symmetry leads to AT $[20,21,26,28]$, this effect is expected to appear in the studied structure having one-side corrugations. Indeed, transmission enhancement at corrugated-side illumination 


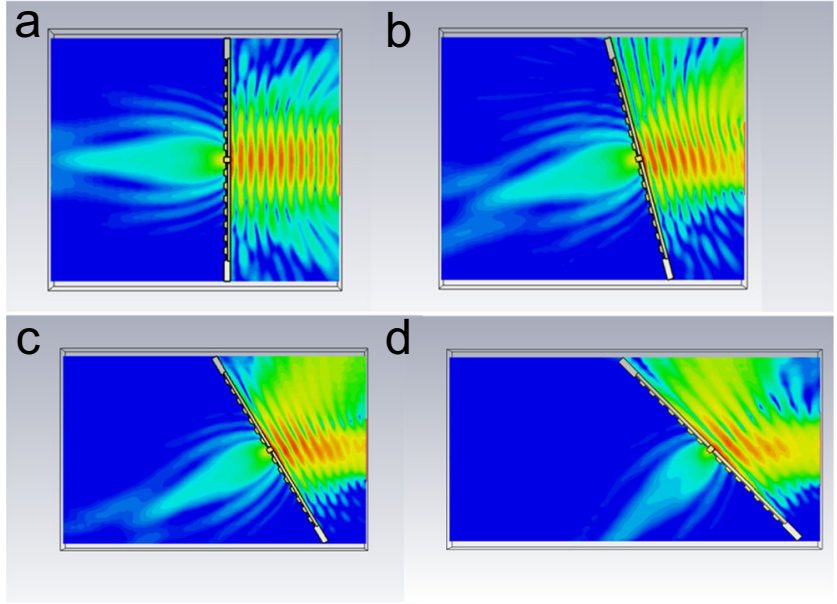

Fig. 6 Magnetic field distribution in $(z, y)$-plane at $\mathbf{a} \theta=0^{\circ}$, b $\theta=15^{\circ}, \mathbf{c} \theta=30^{\circ}, \mathbf{d} \theta=45^{\circ} ; f=13 \mathrm{GHz}$, noncorrugated-side illumination; $a=6.5 \mathrm{~mm}, b=8 \mathrm{~mm} ; t=8 \mathrm{~mm}, p=16 \mathrm{~mm}$, $d=2.4 \mathrm{~mm}$, and $w=p / 2$

can appear due to the effects of spoof SPs at the incidence interface. However, there should be no angular selectivity in the exit half-space, because it is bounded now by the noncorrugated interface, on which spoof SPs are not excited. Therefore, even if the transmission is significant for both noncorrugated-side and corrugated-side illumination, strong asymmetry can manifest itself via different spatial distributions at two opposite directions of incidence. This regime is schematically illustrated in Fig. 7. It is similar to the one studied earlier for the structure with a long slit and lamellar corrugations [34].

Figure 8 presents two examples of the electric field distribution at corrugated-side illumination. To understand the appearance of AT, they should be considered together with Fig. 5a, c, which present the results for noncorrugatedside illumination at the same values of $\theta$. In Fig. 8, none of the angles has an obvious preference for the outgoing radiation, so that the field distributions at the corrugatedside and the noncorrugated-side illumination strongly differ from each other, i.e., asymmetry is well pronounced. This difference can be easily seen from the comparison of Fig. 5a with Fig. 8a, and Fig. 5c with Fig. 8b. It is similar for all other considered angles. Clearly, the field distributions are similar for corrugated-side illumination, but no beam-type collimation occurs in this case. Thus, asymmetry in transmission is connected with one-way beam-type collimation. In turn, it is related to the one-side excitation of spoof SPs. In such a manner, AT and collimation are connected to each other. It can be easily seen that they represent two sides of the same phenomenon that combines the spatial separation of the processes of formation of the field distribution in two half-spaces, which is achieved due to the
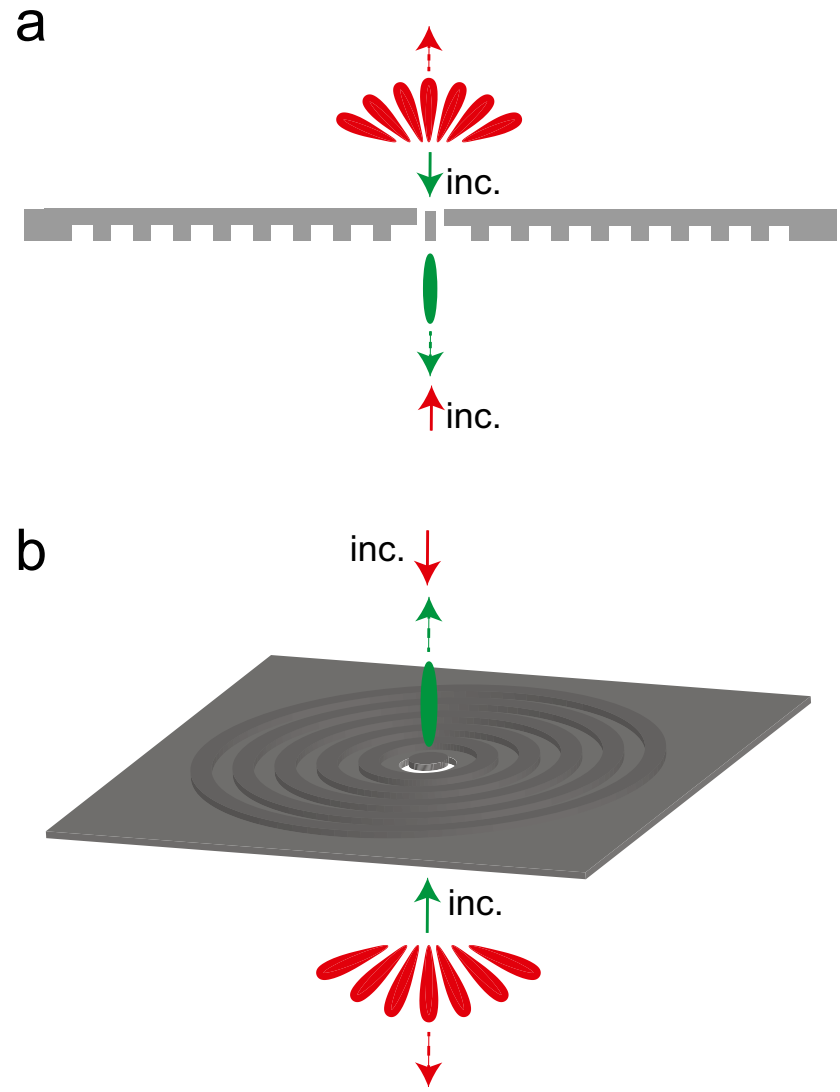

Fig. 7 Schematic illustrating asymmetry in transmission at corrugated-side and noncorrugated-side illumination (red and green colors, respectively) for the structure with an annular hole surrounded by the concentric grooves. Field distributions in the exit half-space at corrugated-side and noncorrugated-side illuminations are different, while beam shaping occurs only in the latter case

subwavelength hole, with one-side excitation of spoof SPs. Since collimation only needs spoof SPs at the exit interface, it does not need the structural asymmetry and, hence, asymmetry in transmission. However, AT in the studied structures needs spoof SPs and collimation at one side only. Thus, either one-side corrugations or different corrugations

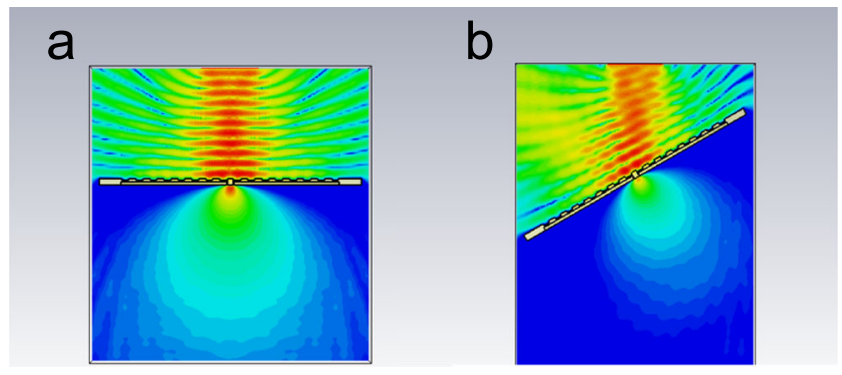

Fig. 8 Electric field distribution in $(x, z)$-plane at $\mathbf{a} \theta=0^{\circ}$ and b $\theta=30^{\circ} ; f=13 \mathrm{GHz}$, corrugated-side illumination; $a=6.5 \mathrm{~mm}$, $b=8 \mathrm{~mm} ; t=8 \mathrm{~mm}, p=16 \mathrm{~mm}, d=2.4 \mathrm{~mm}$, and $w=p / 2$ 
at the two sides can be used. Co-existence of collimation and AT in one structure can also be considered from the multifunctionality perspective $[26,44,45]$.

\section{Independent Processes in Structures with Two-Side Symmetric Corrugations}

As has been demonstrated in "Collimation and Asymmetry in Transmission," AT is impossible without broken structural symmetry, while collimation only needs the specific properties of spoof SPs at the exit interface. Thus, beaming and collimation like the ones in "Collimation and Asymmetry in Transmission" can be possible in the gratings with the same corrugations at two sides. As an example, Fig. 9 presents the electric field distribution just at three selected (among multiple studied) values of $\theta$. It is seen that the radiation shaping in the exit half-space is very similar to Fig. 5 . Direction of the outgoing beam is (nearly) normal in a wide range of the angles, i.e., from 0 to $75^{\circ}$.

Hence, the results in Fig. 9 confirm the assumption that the incidence interface should not affect the shaping in the exit half-space. All the features related to collimation,

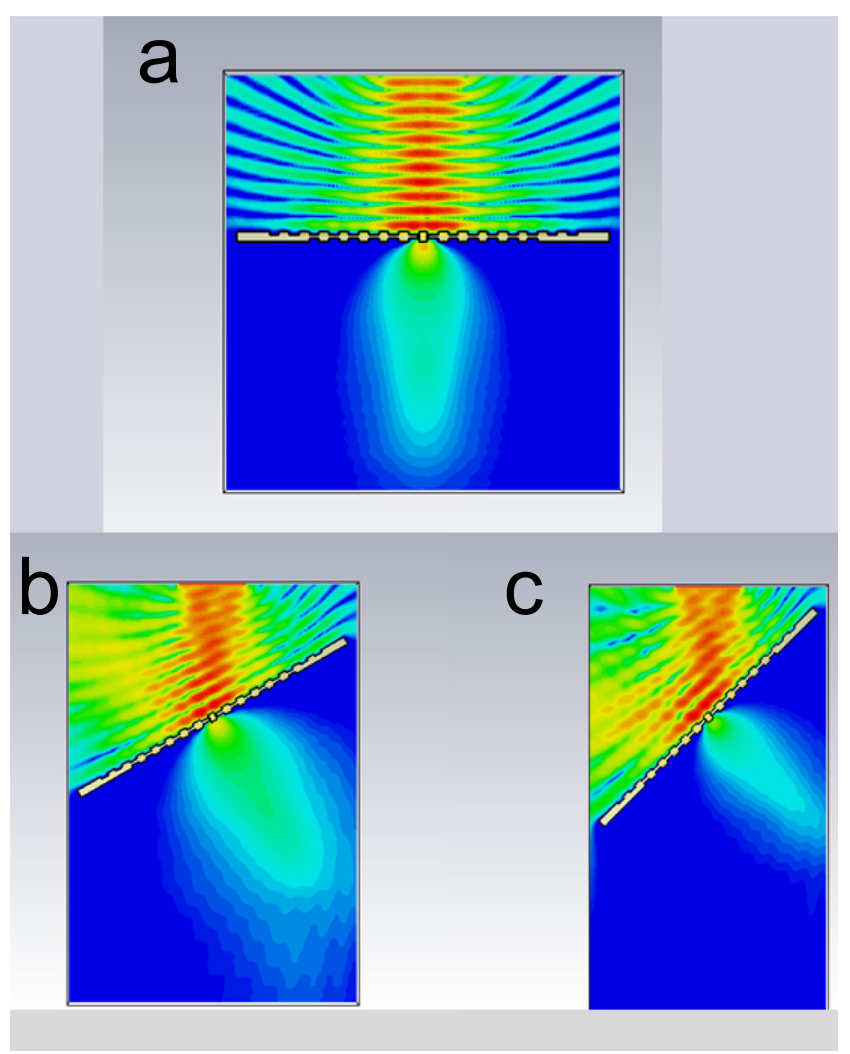

Fig. 9 Electric field distribution in $(x, z)$-plane at $\mathbf{a} \theta=0^{\circ}, \mathbf{b} \theta=30^{\circ}$, c $\theta=45^{\circ} ; f=13 \mathrm{GHz}$, for the structure with two-side corrugations; $a=6.5 \mathrm{~mm}, b=8 \mathrm{~mm} ; t=8 \mathrm{~mm}, p=16 \mathrm{~mm}, d=2.4 \mathrm{~mm}$, and $w=p / 2$ which were discussed above for the structures with one-side corrugations, occur in the studied structure with symmetric two-side corrugations. However, now it does not matter which interface is illuminated. In particular, multiple waves, being simultaneously incident from one half-space at different $\theta$ and the same frequency, may contribute to the same outgoing beam in the other half-space, leading to collimation. Indeed, the linearity of the Maxwell equations enables superposition, so that having results for different $\theta$ is sufficient to predict the field distributions in the case when several waves are simultaneously incident from different directions. The contributions from (a big part of) the whole cone can be collected, similarly to the structures studied in "Collimation and Asymmetry in Transmission."

Using the obtained results (shown and not shown) along with the superposition principle, we conclude that two simultaneous but independent incidence-transmissionreflection wave processes are possible in the structures with two-side corrugations at certain conditions. This scenario is illustrated in Fig. 10 by the schematic being in coincidence with the numerical data. The wave incident from the upper half-space creates the reflected and transmitted waves (group 2), which do not interfere with the reflected and transmitted waves created by the wave incident in the opposite direction (group 1), since all the waves belonging to the group 1 have different propagation directions than the waves of the group 2. The directions of the incident and reflected waves at the incidence from one side do not coincide with the direction of the wave transmitted at the incidence from the opposite side. So, it is obvious that two wave processes initiated by the two different incident waves are independent of each other. Spatial separation and, thus, independence of the two processes are possible due to the shaping of outgoing radiation for one group, so that there is no significant radiation in the incidence and reflection directions for the other group. One can see in Fig. 10 that this is true for nonzero $\theta$. For zero $\theta$, the direction of the wave reflected at the incidence from one side of the structure

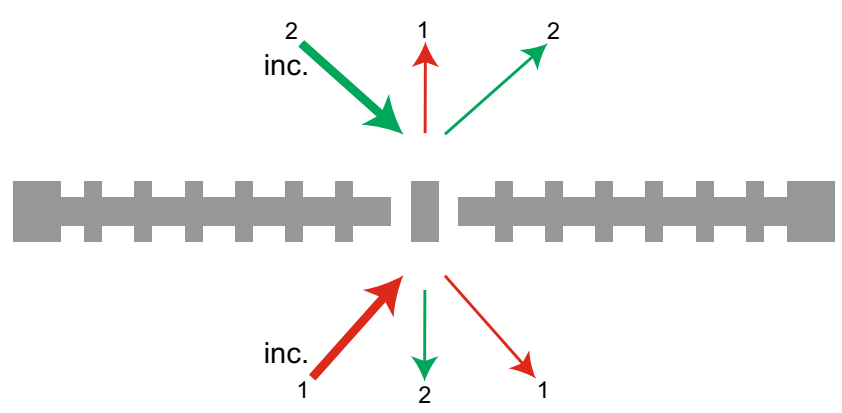

Fig. 10 Schematic illustrating two independent incidencetransmission-reflection processes for the structure with symmetric (two-side) concentric grooves. Directions of the waves belonging to group 1 and group 2 are shown in red and green color, respectively 
will be the same as the direction of the wave transmitted at the incidence from the opposite side, and independence of two wave processes is not possible anymore.

In fact, the requirement of two incidence directions being opposite, which is used in the AT studies, can be mitigated for a symmetric grating with two-side corrugations in the case of two independent collimation processes. One can assume that for the angle of incidence from the upper halfspace, $\theta_{u}$, and for the angle of incidence from the lower half-space, $\theta_{l}$, we have $\theta_{u}>\theta_{0}$ and $\theta_{l}>\theta_{0}$, where $\left|\theta_{0}\right|>0$ is chosen so that interference of the waves belonging to group 1 and group 2 is avoided.

In line with the obtained results and superposition principle, multiple waves which are simultaneously incident from the upper half-space at $\theta_{u}>\theta_{0}$ contribute to the same (collimated) beam in the lower half-space. In turn, the multiple waves which are simultaneously incident from the lower half-space at $\theta_{l}>\theta_{0}$ contribute to the same beam in the upper half-space. Furthermore, the sets of the incidence angle values for the waves incident from the different halfspaces may be completely the same, partially the same, or completely different. Regardless of this, there will be no interference of the waves connected with two processes, i.e., those ones belonging to group 1 and group 2 . In such a way, two collimation processes can be spatially separated.

The obtained results show that collimation of the waves incident at different angles and independence of two processes in the studied structures with two-side corrugations are immune to wide-range variations of $t$ (not shown). It is noteworthy that two independent incidencetransmission-reflection processes have earlier been considered in photonic-crystal-based structures $[20,46]$. Two independent off-axis beaming processes with collimation capability have earlier been studied in thin metallic gratings with a single long slit and different lamellar corrugations at the left and the right side of the exit interface [16]. However, the possibility of two independent collimation processes in three-dimensional structures, like the ones studied here, has not been studied and utilized up to now.

\section{Conclusion}

To summarize, we studied connection between collimation, asymmetric transmission (AT), and independence of two incidence-transmission-reflection processes in the microwave structures with a single-centered annular aperture surrounded by concentric grooves. The use of the annular hole allows to enhance transmission as compared to the circular hole of the same radial size, while keeping the capability of spatial separation of two half-spaces at two sides of the structure. As expected, spatial distribution in each half-space mainly depends on the properties of the bounding interface, also when it may support spoof SPs enabling the radiation shaping. As a result, the incident waves arriving at different angles, i.e., from 0 to $75^{\circ}$, contribute to the same outgoing beam. Hence, the beam sorting in terms of incidence angle is prevented. Collimation is demonstrated in both cases of one-side (asymmetric) and two-side (symmetric) corrugations. Absence of corrugations on one of the sides is sufficient to obtain asymmetry in transmission, even if transmission is significant for two opposite incidence directions. As follows from the obtained results, AT appears as the common effect of separation of field distribution effects in the incidence half-space and the exit half-space due to the subwavelength hole, and the radiation shaping due to the spoof SPs at the exit-side interface. In the studied structures, collimation is possible without AT, while AT is impossible without collimation. For the structures with two-side corrugations, collimation enables two simultaneous processes, which are independent of each other, because propagation directions of all the waves are different and shaping of the outgoing radiation takes place. Moreover, two independent collimation processes are possible, to which different sets of the incidence angles do contribute. The studied structures can be used for communication and sensing applications at microwave frequencies, and may serve as prototypes for future advanced devices in millimeter, terahertz, and optical wavelength ranges that use the same or similar physical scenarios.

Funding Information This work was financially supported by the Narodowe Centrum Nauki (NCN), Poland (DEC-2015/17/B/ST3/00118Metasel); Academy of Finland (COMPETITIVE FUNDING TO STRENGTHEN UNIVERSITY RESEARCH PROFILES, decision number 301820); EU Horizon-2020 via Marie Sklodowska-Curie IF program (grant no. 708200 - ADVANTA). E.O. received partial financial support from the Turkish Academy of Sciences.

\section{References}

1. Ebbesen TW, Lezec HJ, Ghaemi HF, Thio T, Wolff PA (1998) Extraordinary optical transmission through sub-wavelength hole arrays. Nature 391:667-669

2. Thio T, Pellerin KM, Linke RA, Lezec HJ, Ebbesen TW (2001) Enhanced light transmission through a single subwavelength aperture. Opt Lett 26:1972-1974

3. Caglayan H, Bulu I, Ozbay E (2005) Extraordinary gratingcoupled microwave transmission through a subwavelength annular aperture. Opt Express 13:1666-1671

4. Caglayan H, Bulu I, Ekmel O (2006) Plasmonic structures with extraordinary transmission and highly directional beaming properties. Microwave Opt Technol Lett 48:2491-2496

5. Lezec HJ, Degiron A, Devaux E, Linke RA, Martin-Moreno L, Garcia-Vidal FJ, Ebbesen TW (2002) Beaming light from a subwavelength aperture. Science 297:820-822

6. Degiron A, Ebbesen TW (2004) Analysis of the transmission process through single apertures surrounded by periodic corrugations. Opt Express 12:3694-3700 
7. Li Z, Caglayan H, Colak E, Ozbay E (2008) Enhanced transmission and directivity from metallic subwavelength apertures with nonuniform and nonperiodic grooves. Appl Phys Lett 92:011128

8. Yu N, Capasso F (2010) Wavefront engineering for mid-infrared and terahertz quantum cascade lasers. J Opt Soc Am B 27:B18-B35

9. Caglayan H, Bulu I, Ozbay E (2006) Beaming of electromagnetic waves emitted through a subwavelength annular aperture. J Opt Soc Am B 23:419-422

10. Kim S, Kim H, Lim Y, Lee B (2007) Off-axis directional beaming of optical field diffracted by a single subwavelength metal slit with asymmetric dielectric surface gratings. Appl Phys Lett 90:051113

11. Lin DZ, Cheng TD, Chang CK, Yeh JT, Liu JM, Yeh CS, Lee CK (2007) Directional light beaming control by a subwavelength asymmetric surface structure. Opt Express 15:2585-2591

12. Caglayan H, Bulu I, Ozbay E (2008) Off-axis beaming from subwavelength apertures. J Appl Phys 104:073108

13. Caglayan H, Bulu I, Ozbay E (2009) Observation of offaxis directional beaming via subwavelength asymmetric metallic gratings. J Phys D: Appl Phys 42:045105

14. Lee Y, Hoshino K, Alu A, Zhang X (2013) Tunable directive radiation of surface-plasmon diffraction gratings. Opt Express 21:27482756

15. Zhou Y, Lu MH, Feng L, Ni X, Chen YF, Zhu YY, Ming NB (2010) Acoustic surface evanescent wave and its dominant contribution to extraordinary acoustic transmission and collimation of sound. Phys Rev Lett 104:164301

16. Cakmakyapan S, Serebryannikov AE, Caglayan H, Ozbay E (2012) Spoof-plasmon relevant one-way collimation and multiplexing at beaming from a slit in metallic grating. Opt Express 20:26636-26648

17. Panaretos AH, Werner DH (2016) Leaky wave lenses for spoof plasmon collimation. Opt Express 24:14654-14671

18. Serebryannikov AE, Magath T, Schuenemann K (2006) Bragg transmittance of s-polarized waves through finite-thickness photonic crystals with a periodically corrugated interface. Phys Rev E 74:066607

19. Serebryannikov AE, Lakhtakia A (2013) Switchable photonic crystal grating diode using coherent atomic gas. Microwave Opt Technol Lett 55:1248-1250

20. Serebryannikov AE, Cakmak AO, Ozbay E (2012) Multichannel optical diode with unidirectional diffraction relevant total transmission. Opt Express 20:14980-14990

21. Lockyear MJ, Hibbins AP, White KR, Sambles JR (2006) Oneway diffraction grating. Phys Rev E 74:056611

22. Xu T, Lezec HJ (2014) Visible-frequency asymmetric transmission devices incorporating a hyperbolic metamaterial. Nat Commun 5:4141

23. Monnai Y, Jahn D, Withayachumnankul W, Koch M, Shinoda H (2015) Terahertz plasmonic Bessel beamformer. Appl Phys Lett 106:021101

24. Beaskoetxea U, Maci S, Navarro-Cia M, Beruete M (2017) 3Dprinted $96 \mathrm{GHz}$ bull's-eye antenna with off-axis beaming. IEEE Trans Antennas Propag 65:17-25

25. Sattari H, Rashed AR, Ozbay E, Caglayan H (2017) Bright off-axis directional emission with plasmonic corrugations. Opt Express 25:30827-30842

26. Mutlu M, Cakmakyapan S, Serebryannikov AE, Ozbay E (2013) One-way reciprocal spoof surface plasmons and relevant reversible diodelike beaming. Phys Rev B 87:205123

27. Stolarek M, Yavorskiy D, Kotyński R, Rodríguez CJZ, Lusakowski J, Szoplik T (2013) Asymmetric transmission of terahertz radiation through a double grating. Opt Lett 38:839-841
28. Serebryannikov AE, Ozbay E, Nojima S (2014) Asymmetric transmission of terahertz waves using polar dielectrics. Opt Express 22:3075-3088

29. Wang C, Zhong XL, Li ZY (2012) Linear and passive silicon optical isolator. Sci Rep 2:674

30. Gundogdu FT, Serebryannikov AE, Cakmak AO, Ozbay E (2015) Asymmetric transmission in prisms using structures and materials with isotropic-type dispersion. Opt Express 23:2412024132

31. Menzel C, Helgert C, Rockstuhl C, Kley EB, Tunnermann A, Pertsch T, Lederer F (2010) Asymmetric Transmission of linearly polarized light at optical metamaterials. Phys Rev Lett $104: 253902$

32. Serebryannikov AE, Beruete M, Mutlu M, Ozbay E (2015) Multiband one-way polarization conversion in complementary split-ring resonator based structures by combining chirality and tunneling. Opt Express 23:13517-13529

33. Yao Y, Liu H, Wang Y, Li Y, Song B, Wang RP, Wu W (2016) Nanoimprint-defined, large-area meta-surfaces for unidirectional optical transmission with superior extinction in the visible-toinfrared range. Opt Express 24:15362-15372

34. Cakmakyapan S, Serebryannikov AE, Caglayan H, Ozbay E (2010) One-way transmission through the subwavelength slit in nonsymmetric metallic gratings. Opt Lett 35:2597-2599

35. Cakmakyapan S, Caglayan H, Serebryannikov AE, Ozbay E (2011) Experimental validation of strong directional selectivity in nonsymmetric metallic gratings with a subwavelength slit. Appl Phys Lett 98:051103

36. Baida FI, Van Labeke D (2003) Three-dimensional structures for enhanced transmission through a metallic film: Annular aperture arrays. Phys Rev B 67:155314

37. Lockyear MJ, Hibbins AP, Sambles JR, Lawrence CR (2005) Microwave transmission through a single subwavelength annular aperture in a metal plate. Phys Rev Lett 94:193902

38. Garcia-Vidal FJ, Martin-Moreno L, Pendry JB (2005) Surfaces with holes in them: new plasmonic metamaterials. J Opt A: Pure Appl Opt 7:S97-S101

39. Chen J, Li Z, Yue S, Gong Q (2010) Efficient unidirectional generation of surface plasmon polaritons with asymmetric singlenanoslit. Appl Phys Lett 97:041113

40. Raether H (1988) Surface plasmons on gratings. In: Surface plasmons on smooth and rough surfaces and on gratings. Springer, Berlin, pp 91-116

41. Teng YY, Stern EA (1967) Plasma radiation from metal grating surfaces. Phys Rev Lett 19:511-514

42. Thio T, Lezec HJ, Ebbesen TW, Pellerin KM, Lewen GD, Nahata A, Linke RA (2002) Giant optical transmission of sub-wavelength apertures: physics and applications. Nanotechnology 13:429-432

43. Baida FI, Van Labeke D, Granet G, Moreau A, Belkhir A (2004) Origin of the super-enhanced light transmission through a 2-D metallic annular aperture array: a study of photonic bands. Appl Phys B 79:1-8

44. Cai T, Wang GM, Xu HX, Tang SW, Li H, Liang JG, Zhuang YQ (2018) Bifunctional Pancharatnam-Berry metasurface with high efficiency helicity dependent transmissions and reflections. Ann Physik (Berlin) 530:1700321

45. Jang T, Youn H, Shin YJ, Guo LJ (2014) Transparent and flexible polarization-independent microwave broadband absorber. ACS Photonics 1:279-284

46. Gupta MM, Medhekar S (2014) A versatile optical junction using photonic band-gap guidance and self collimation. Appl Phys Lett 105:131104 